\title{
Asymptomatic Bacteriuria in pregnant women attending a tertiary hospital in northern India: prevalence, risk factors, causative organisms and antimicrobial sensitivity pattern
}

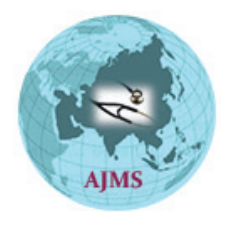

\author{
Harman Multani' ${ }^{1}$, Humaira Bashir ${ }^{2}$, Seema Aleem ${ }^{3}$ \\ ${ }^{1}$ Senior Resident, Department of Microbiology, Government Medical College, Jammu, J \& K, India, ${ }^{2}$ Lecturer, ${ }^{3}$ Senior \\ Resident, Department of Microbiology, Government Medical College, Srinagar, Srinagar, J \& K, India
}

Background: Asymptomatic bacteriuria is common and can lead to adverse foetal and maternal outcomes. Aims and Objective: The current study aimed to estimate the prevalence of asymptomatic bacteriuria ( $A B U$ ), its associated risk factors, bacterial isolates and antimicrobial sensitivity pattern in pregnant women attending Outpatient department (services in a tertiary hospital in India. Materials and Methods: This was a hospital-based cross-sectional study among asymptomatic women coming for routine antenatal check-ups to obstetrics OPD of a teaching hospital in Northern India. Samples were collected, processed and antimicrobial sensitivity done as per Clinical and Laboratory Standards Institute (CLSI) guidelines. Data was analysed and p value below 0.05 was considered as statistically significant. Results: Overall 247 pregnant women were included in the study with a mean age of 25.9 \pm 3.1 . The overall prevalence of asymptomatic bacteriuria was $18.6 \%$. The most common isolates responsible for ABU were E. Coli (48\%), S Aureus (23\%), K. pneumonia (12\%), Pseudomonas (6\%), Enterococcus (6\%), Proteus (5\%). Gram-negative isolates were mostly sensitive to Fosfomycin, Meropenem, Piperacillin + Tazobactum whereas gram-positive isolates were usually sensitive to Fosfomycin, Vancomycin and Linezolid Most isolates were resistant to Co-amoxiclav, Ciprofloxacin, and Nitrofurantoin. Conclusion: Considering the high prevalence of $\mathrm{ABU}$ and its association with adverse maternal and foetal outcomes, urine culture should be included in routine investigations being offered to pregnant women. Gram-negative bacteria were the primary causative agents with Fosfomycin, Meropenem and Ceftazidime showing high level of efficacy against the pathogens.

Key words: Antibiotics; Bacteriuria; Culture; Fosfomycin; Sensitivity
Access this article online

\section{Website:}

http://nepjol.info/index.php/AJMS DOI: 10.3126/ajms.v12i9.37039 E-ISSN: 2091-0576 P-ISSN: 2467-9100

Copyright (c) 2021 Asian Journal of Medical Sciences

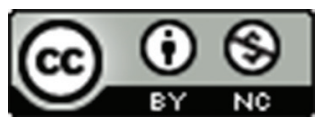

This work is licensed under a Creative Commons Attribution-NonCommercial 4.0 International License.

\section{INTRODUCTION}

Asymptomatic bacteriuria $(\mathrm{ABU})$ is defined as the presence of bacteria in the urine of a person without signs or symptoms of a urinary tract infection. ${ }^{1}$ As the person is asymptomatic, asymptomatic bacteriuria can only be diagnosed by urine cultures. Laboratory criteria for its diagnosis are colony forming units (CFU) of 100,000 per $\mathrm{ml}$ of a midstream clean catch urine sample., ${ }^{2,3}$ Current guidelines do not recommend routine screening for asymptomatic bacteriuria as it has not been associated with an increase in morbidity or mortality in general population. Pregnant women form an important exception in which untreated asymptomatic bacteriuria can cause adverse maternal and foetal outcomes. ${ }^{4} \mathrm{ABU}$ has been associated with acute pyelonephritis in pregnant women with a number of studies finding increased risk of premature births, low birth weight or both. Higher prevalence, as well as higher risk of morbidity due to $\mathrm{ABU}$ in pregnancy can be related to the hormonal, anatomical, mechanical and immunological changes associated with the pregnancy. ${ }^{5}$ In view of these dangers screening for $\mathrm{ABU}$ and prompt 
treatment of pregnant women with $\mathrm{ABU}$ is a standard practice in antenatal care.

The current study was planned to estimate the prevalence of $\mathrm{ABU}$, its risk factors, microbiological profile and antibiotic sensitivity profile of bacteria associated with ABU. The results from this study can guide in decision making related to screening for $\mathrm{ABU}$ and also guide in the selection of proper antibiotics.

The objectives of this study were to estimate the prevalence of $\mathrm{ABU}$, its associated risk factors, microbiological profile and antibiotic sensitivity profile in pregnant women attending ante-natal OPD in a tertiary care hospital.

\section{MATERIALS AND METHODS}

\section{Study design, setting and area}

The current study was a cross-sectional study conducted among ante-natal women attending an obstetric clinic at a teaching hospital in Haryana state of northern India. The hospital has a high footfall for OPD services with a large number of patients visiting the hospital for routine antenatal services. The routine antenatal services are provided by residents and faculty from the department of gynecology and obstetrics.

\section{Study participants}

All pregnant women who came for an antenatal check-up between January 2016 to April 2016 were approached and explained about the purpose of the study and their consent sought for participation in the study. Women with symptoms of UTI, fever and women with history of urolithiasis, urological surgery urogenital fistula were excluded from the study. In addition women who were currently taking antimicrobials or had taken antimicrobials in last two weeks were excluded from the study. Only women who provided informed consent were included in the study. A pretested, self-administered schedule was used to collect socio-demographic information, risk factors, $\mathrm{h} / \mathrm{o}$ gestational diabetes, $\mathrm{h} / \mathrm{o}$ hypertension, previous and current obstetric history and previous medical/surgical history. Following the completion of the questionnaire, each subject was explained about the process for collection of a urine sample. The subjects were directed towards a female washroom with the provision for handwashing to avoid contamination of samples.

\section{Collection of urine samples}

Each pregnant woman was provided with a sterile and labelled screw capped collection vial. Process of sample collection was explained to reduce chances of contamination. $10-15 \mathrm{ml}$ of midstream urine sample was collected. The samples were processed within 1-2 hours of collection for analysis. A routine microscopy was not performed and the samples were directly processed for culture and sensitivity.

\section{Processing and isolation of samples}

Urine samples were inoculated and streaked with the help of heat flamed standard wire loop (delivering $0.001 \mathrm{ml}$ ) on the culture plate. The culture media used for isolation were cysteine-Lactose Electrolyte-Deficient, blood and chocolate agar plates. The plates were incubated aerobically at $37 \mathrm{c}$ for $24 \mathrm{hrs}$ and then examined. Plates with significant growth (at least $100.000 \mathrm{CFU} / \mathrm{ml}$ ) were considered to be significant and further analysed for microbial identification and drug sensitivity. After incubation, subcultures and biochemical tests on MacConkey agar, Mannitol salt agar and 5\% sheep blood agar were done for identification. Repeat cultures were not performed for samples with significant growth.

\section{Antimicrobial susceptibility testing}

Antimicrobial susceptibility testing was performed using Kirby-Bauer (KB) disk diffusion test and interpreted using CLSI and European Committee on Antimicrobial Susceptibility Testing (EUCAST) guidelines. ${ }^{6,78}$ The inoculated culture plates were left at room temperature and disks impregnated with Nitrofurantoin, Fosfomycin, Co-amoxiclav, Ciprofloxacin, Ceftazidime, Piperacillin + Tazobactum, Meropenem, Amikacin, Vancomycin, Linezolid, Penicillin. The culture plates were then inoculated for 24 hours at 37 degrees. They were classified as susceptible and resistant by measurement of inhibition zones using rulers and as per CLSI guidelines.

\section{Statistical methods}

Data was first entered in Microsoft excel and then exported to SPSS for analysis. Data was summarized using frequency tables and cross tabs. $\mathrm{p}$ value below 0.05 was considered statistically significant.

\section{RESULTS}

\section{Socio-demographic characteristics}

A total of 247 pregnant women without signs and symptoms of UTI were included in the study. The age of subjects ranged from 19 years to 34 years with a mean age of $25.9 \pm 3.1$. Almost $62 \%$ of participants belonged to rural areas. Almost $1 / 3^{\text {rd }}$ of participants worked as farmers and another $1 / 3^{\text {rd }}$ were home makers. Highest proportion of participants belonged to socio-economic class 1 and $2 .{ }^{9}$ Half of the participants were in third trimester of their pregnancy and half of the study participants were anaemic ( $\mathrm{Hb}$ level less than $11 \mathrm{~g} / \mathrm{dl})$. The details are depicted in Table 1. 
Of the study participants, 46 (18.6\%) had significant Bacteriuria. Among these one urine sample $(2.1 \%)$ showed mixed bacterial growth with the rest demonstrating single bacterial growth.

Gram-negative bacteria were the predominant organism. The most common isolated organism was E. coli which contributed to $48 \%$ of isolates. Among gram-negative organisms, it was followed by K. pneumonia, Pseudomonas aeruginosa and Proteus mirabilis which contributed to $12.5 \%, 6.2 \%$ and $4 \%$ isolates respectively. Gram positive organisms contributed to $29 \%$ of isolates with staphylococcus aureus being the most common organism followed by Enterococcus faecalis.

Antimicrobial sensitivity of Gram-negative isolates In this study, Gram negative isolates were sensitive to Fosfomycin, Meropenem, Piperacillin + Tazobactum. These were mostly resistant to Co-amoxiclav, Ciprofloxacin.
Drug sensitivity profiles of individual bacteria are depicted in Table 2.

Antimicrobial sensitivity of Gram-positive isolates For Gram positive isolates which were mostly contributed by S. Aureus, the isolates were mostly resistant to Nitrofurantoin, Co-amoxiclav, Ciprofloxacin, and Penicillin. The isolates were mostly sensitive to Fosfomycin, Vancomycin and Linezolid. Drug sensitivity profiles of individual bacteria are depicted in Table 2.

\section{DISCUSSION}

Appropriate diagnoses and management of $\mathrm{ABU}$ is important as untreated $\mathrm{ABU}$ can lead to adverse maternal and foetal outcomes. In this study the prevalence of $\mathrm{ABU}$ was estimated at $18.6 \%$. the results are comparable to few studies

\begin{tabular}{|c|c|c|c|c|c|}
\hline Variables & Positive No & Negative No & Total No & $\%$ Positive & P Value \\
\hline $\mathrm{ABU}$ & 46 & 201 & 247 & 18.6 & \\
\hline \multicolumn{6}{|l|}{ Age } \\
\hline $19-22$ & 8 & 39 & 47 & 17.0 & $X 2=0.162$ \\
\hline $23-26$ & 17 & 74 & 91 & 18.7 & $p=.098$ \\
\hline $27-30$ & 15 & 65 & 80 & 18.5 & \\
\hline $31-34$ & 6 & 23 & 29 & 21.4 & \\
\hline \multicolumn{6}{|l|}{ Hb Level } \\
\hline Normal & 19 & 97 & 116 & 16.4 & $X 2=0.727$ \\
\hline Anaemia & 27 & 104 & 131 & 20.6 & $p=.394$ \\
\hline \multicolumn{6}{|l|}{ Parity } \\
\hline Nulliparous & 14 & 84 & 98 & 14.3 & $X 2=2.19$ \\
\hline Primiparous & 19 & 74 & 93 & 20.4 & $p=.333$ \\
\hline Multi & 13 & 43 & 56 & 23.2 & \\
\hline \multicolumn{6}{|l|}{ Gestational period } \\
\hline $1^{\text {st }}$ & 6 & 39 & 45 & 13.3 & $X 2=1.11$ \\
\hline $2^{\text {nd }}$ & 14 & 61 & 75 & 18.7 & $p=.571$ \\
\hline $3^{\text {rd }}$ & 26 & 101 & 127 & 20.5 & \\
\hline \multicolumn{6}{|l|}{ Education } \\
\hline Illiterate & 12 & 52 & 64 & 18.8 & $X 2=0.021$ \\
\hline Literate & 5 & 21 & 26 & 19.2 & $p=.999$ \\
\hline Primary & 17 & 75 & 92 & 18.5 & \\
\hline Secondary & 7 & 30 & 37 & 18.9 & \\
\hline Higher education & 5 & 23 & 28 & 17.9 & \\
\hline \multicolumn{6}{|l|}{ Residence } \\
\hline Rural & 32 & 121 & 153 & 20.9 & $p=.237$ \\
\hline Urban & 14 & 80 & 94 & 14.9 & \\
\hline \multicolumn{6}{|l|}{ Occupation } \\
\hline Farmer & 21 & 73 & 94 & 22.3 & $X 2=0.727$ \\
\hline Home maker & 13 & 67 & 80 & 16.3 & $p=.394$ \\
\hline Salaried (orqanized sector) & 5 & 26 & 31 & 16.1 & \\
\hline Salaried (un-organized sector) & 7 & 35 & 42 & 16.7 & \\
\hline \multicolumn{6}{|l|}{ Socioeconomic status } \\
\hline (low) 1 & 19 & 69 & 88 & 21.6 & $X 2=1.63$ \\
\hline 2 & 16 & 65 & 81 & 19.8 & $p=.651$ \\
\hline 3 & 6 & 36 & 42 & 14.3 & \\
\hline 4 & 5 & 31 & 36 & 13.9 & \\
\hline \multicolumn{6}{|l|}{$\mathrm{H} / \mathrm{O}$ of UTI } \\
\hline Yes & 12 & 27 & 39 & 30.7 & $P=0.04$ \\
\hline No & 34 & 174 & 208 & 16.3 & \\
\hline \multicolumn{6}{|l|}{$\mathrm{H} / \mathrm{O}$ catheterization } \\
\hline Yes & 14 & 34 & 48 & 41.1 & $X 2=4.3$ \\
\hline NO & 32 & 167 & 199 & 16.0 & $p=.03$ \\
\hline
\end{tabular}


Table 2: Table depicting antimicrobial sensitivity of microbial isolates

\begin{tabular}{|c|c|c|c|c|c|c|c|c|c|}
\hline \multirow{2}{*}{$\begin{array}{l}\text { Bacterial isolates } \\
\text { Gram negative }\end{array}$} & \multicolumn{9}{|c|}{ No. of strains sensitive to antibiotics (\%) } \\
\hline & No & NIT & FO & AMC & CIP & CTZ & PIT & MRP & AK \\
\hline E. coli & 23 & 87 & 91 & 52 & 52 & 78 & 87 & 83 & 78 \\
\hline K. Pneumoniae & 6 & 67 & 83 & 33 & 33 & 67 & 83 & 67 & 83 \\
\hline P. Aeruginosa & 3 & 66 & 100 & 33 & 33 & 100 & 100 & 66 & 66 \\
\hline Proteus & 2 & 0 & 100 & 50 & 0 & 100 & 100 & 100 & 50 \\
\hline Total & 34 & & & & & & & & \\
\hline Gram positive & No. & NIT & FO & AC & CF & VM & $\mathrm{LZ}$ & PE & \\
\hline S. Aureus & 11 & 64 & 91 & 54 & 36 & 82 & 100 & 18 & \\
\hline Enterococcus & 3 & 100 & 100 & 100 & 0 & 100 & 100 & 66 & \\
\hline Total & 14 & & & & & & & & \\
\hline
\end{tabular}

Nitrofurantoin (NIT), Fosfomycin (FO), Co-amoxiclav (AMC), Ciprofloxacin (CIP), Ceftazidime (CTZ, Piperacillin + Tazobactum (PIT), Meropenem (MRP), Amikacin (AK), ), CoAmoxiclav(AC), Cefoxitin (CF), Vancomycin (VM), Linezolid (LZ), Penicillin (PE)

done in north India. ${ }^{10,11}$ Though the prevalence estimated is higher than few studies conducted in India which may be due to differences in composition of the population being catered to by this hospital. ${ }^{12,13}$ In the current study, ABU was not found to have a statistically significant relation with age, haemoglobin level, parity, gestational period, education, residence or socioeconomic status even though different studies have found an association with some of these factors. This could be due to differences in population characteristics in terms of education and hygiene.

Asymptomatic bacteriuria was found to be significantly related with a previous history of urinary tract infection and catheterization. The same finding has been found by multiple other studies. ${ }^{14,15}$ This finding reinforces the need for ensuring that all aseptic precautions are followed during catheterization, particularly during childbirth. Though there was no significant association with the parity of women which could be due to small sample size for identifying any relation with parity.

Gram-negative bacteria mostly contributed by E Coli were the predominant organisms which contributed to $70 \%$ of isolates. The bacteria responsible for $\mathrm{ABU}$ are primarily of faecal origins which colonize the peri-urethral area. This finding is similar to multiple other studies as most studies have found E. Coli to be the most common pathogen responsible for ABU. ${ }^{12,16,17}$ The second most common pathogen responsible for $\mathrm{ABU}$ in our study was S. Aureus which has also been identified in multiple studies. ${ }^{14,18}$ Among gram-negative organisms, it was followed by K. pneumonia, Pseudomonas and Proteus which contributed to $12.5 \%, 6.2 \%$ and $4 \%$ isolates respectively. Overall Grampositive organisms contributed to $29 \%$ with Enterococcus contributed to the rest of gram-positive isolates.

\section{Sensitivity to antimicrobials}

In this study, gram-negative isolates were mostly sensitive to Fosfomycin, Meropenem, Piperacillin + Tazobactum. These were mostly resistant to Co-amoxiclav, Ciprofloxacin and Nitrofurantoin. Around $2 / 3^{\text {rd }}$ of gram negative isolated were sensitive to cotrimoxazole. This is similar to what has been found in multiple other studies. This can also be due the fact that these antibiotics are commonly prescribed over the counter drugs in India.

For gram positive isolates which were mostly contributed by S. Aureus, the isolates were mostly resistant to Nitrofurantoin, Co-amoxiclav, Cefoxitin, and Penicillin. The isolates were mostly sensitive to Fosfomycin, Vancomycin and Linezolid. Resistance to cefoxitin was used as a maker for Methicillin Resistant Staphylococcus Aureus (MRSA). Around 2/3 ${ }^{\text {rd }}$ of isolates were to Cefoxitin resistant. Development of drug resistance for infections related to pregnancy is a grave problem as it further reduces the available options which are already reduced due to pregnancy.

In conclusion, the overall prevalence of $\mathrm{ABU}$ in our study was $18.6 \%$, the most common agent for ABU was E. Coli followed by S Aureus. Gram-negative isolates were mostly resistant to Co-Amoxiclav, Ciprofloxacin and Nitrofurantoin whereas Gram-positive isolates were mostly resistant to Nitrofurantoin, Co-Amoxiclav, Ciprofloxacin, and Penicillin. Bost groups were mostly sensitive to Fosfomycin and Piperacillin + Tazobactum.

\section{Recommendations}

Considering the high prevalence of $\mathrm{ABU}$ and the fact that untreated cases can have adverse maternal and foetal outcomes, urine culture should be included in the routine investigations being offered to pregnant women. Further studies can be conducted to identify the best time for conducting routine urine cultures in pregnant women.

\section{Limitations of the study}

The study subjects mostly belonged to rural areas so the findings cannot be extrapolated to urban populations and non-agrarian groups.

\section{Ethics statement}

All the subjects who had significant bacteriuria were contacted and explained the result and were advocated to consult their treating doctor. 


\section{ACKNOWLEDGMENT}

The authors would like to thank the whole staff from the department of microbiology and OBGY for their support in the study.

\section{REFERENCES}

1. Foxman B. Urinary Tract Infection Syndromes: Occurrence, Recurrence, Bacteriology, Risk Factors, and Disease Burden. Infect Dis Clin North Am. 2013;28(1):1-13.

https://doi.org/10.1016/j.idc.2013.09.003

2. Lerma EV. Asymptomatic Bacteriuria [Internet]. emedscape. 2017 [cited 2020 Dec 16].

3. Nicolle LE, Gupta K, Bradley SF, Colgan R, DeMuri GP, Drekonja $\mathrm{D}$, et al. Clinical practice guideline for the management of asymptomatic bacteriuria: 2019 update by the Infectious Diseases Society of America. Clinical Infectious Diseases (Oxford University Press). 2019; Vol. 68, [cited 2021 May 30]. E83-E75.

https://doi.org/10.1093/cid/ciz021

4. Wingert A, Pillay J, Sebastianski M, Gates M, Featherstone R, Shave $\mathrm{K}$, et al. Asymptomatic bacteriuria in pregnancy: systematic reviews of screening and treatment effectiveness and patient preferences. BMJ Open. 2019; 9(3): e021347.

https://doi.org/10.1136/bmjopen-2017-021347

5. Nteziyaremye J, Iramiot SJ, Nekaka R, Musaba MW, Wandabwa J, Kisegerwa E, et al. Asymptomatic bacteriuria among pregnant women attending antenatal care at Mbale Hospital, Eastern Uganda. PLoS ONE. 2020;15(3):1-12.

https://doi.org/10.1371/journal.pone.0230523

6. Melvis P Weinstein JSL. The Clinical and Laboratory Standards Institute Subcommittee on Antimicrobial Susceptibility Testing: Background, Organization, Functions, and Processes. J Clin Microbiol. 2020; 58(3):1-7.

https://doi.org/10.1128/JCM.01864-19

7. Sánchez-Bautista A, Coy J, García-Shimizu P and Rodríguez JC. From CLSI to EUCAST guidelines in the interpretation of antimicrobial susceptibility: What is the effect in our setting? Enferm Infecc Microbiol Clin (Engl Ed). 2018;36(4):229-232. https://doi.org/10.1016/j.eimce.2018.02.012

8. Wolfensberger A, Sax H, Weber R, Zbinden R, Kuster SP and Hombach M. Change of antibiotic susceptibility testing guidelines from CLSI to EUCAST: Influence on cumulative hospital antibiograms. PLoS One. 2013;8(11):e79130.

https://doi.org/10.1371/journal.pone.0079130

9. Wani RT. Socioeconomic status scales-modified Kuppuswamy and Udai Pareekh's scale updated for 2019. J Family Med Prim Care. 2019;8(6):1846-1849. https://doi.org/10.4103/jfmpc.jfmpc_288_19

10. Bandyopadhyay S, Thakur JS, Ray P and Kumar R. High prevalence of bacteriuria in pregnancy and its screening methods in north India. Journal of the Indian Medical Association. 2005;103(5):259-266.

11. Lata I, Pradeep $Y$ and Sujata JA. Estimation of the incidence of bacterial vaginosis and other vaginal infections and its consequences on maternal/fetal outcome in pregnant women attending an antenatal clinic in a tertiary care hospital in North India. Indian J Community Med. 2010;35(2):285-289. https://doi.org/10.4103/0970-0218.66855

12. Chunchaiah S, Nagothi NP, Roopakala B and Rangaiah N. A prospective study of prevalence, risk factors, isolates \& antimicrobial sensitivity pattern in Asymptomatic Bacteriuria among Antenatal women in Rajarajeswari Medical College \& Hospital. Indian Journal of Obstetrics and Gynecology Research. 2016;3(3):229.

https://doi.org/10.5958/2394-2754.2016.00052.7

13. Sujatha $R$ and Nawani $M$. Prevalence of asymptomatic bacteriuria and its antibacterial susceptibility pattern among pregnant women attending the antenatal clinic at Kanpur, India. J Clin Diagn Res.2014;8(4):DC01-DC03.

https://doi.org/10.7860/JCDR/2014/6599.4205

14. Ali IE, Gebrecherkos T, Gizachew M and Alemayehu Menberu M. Asymptomatic bacteriuria and antimicrobial susceptibility pattern of the isolates among pregnant women attending Dessie referral hospital, Northeast Ethiopia: A hospital-based cross-sectional study. Turkish Journal of Urology. 2018;44(3):251-260.

https://doi.org/10.5152/tud.2018.07741

15. Rajaratnam A, Baby NM, Kuruvilla TS and Machado S. Diagnosis of asymptomatic bacteriuria and associated risk factors among pregnant women in Mangalore, Karnataka state. J Clin Diagn Res. 2014;8(9):OC23-OC25.

https://doi.org/10.7860/JCDR/2014/8537.4842

16. Gilbert NM, O'brien VP, Hultgren S, Macones G, Lewis WG and Lewis AL. Urinary Tract Infection as a Preventable Cause of Pregnancy Complications: Opportunities, Challenges, and a Global Call to Action. Global Advances in Health and Medicine. 2013;2(5):59-69.

https://doi.org/10.7453/gahmj.2013.061

17. Tadesse S, Kahsay T, Adhanom G, Kahsu G, Legese H, Gwahid A et al. Prevalence, antimicrobial susceptibility profile and predictors of asymptomatic bacteriuria among pregnant women in Adigrat General Hospital, Northern Ethiopia. BMC Research Notes. 2018;11(1). https://doi.org/10.1186/s13104-018-3911-7

18. PT Aernan and EU Umeh GG. Asymptomatic bacteriuria in pregnant women attending a tertiary health care centre in Asymptomatic Bacteriuria (ASB) In Pregnant Women Attending A Tertiary Health Care Centre In Makurdi, Central Nigeria. International Journal of Innovative Research and Advanced Studies. 2016; 3:3-6.

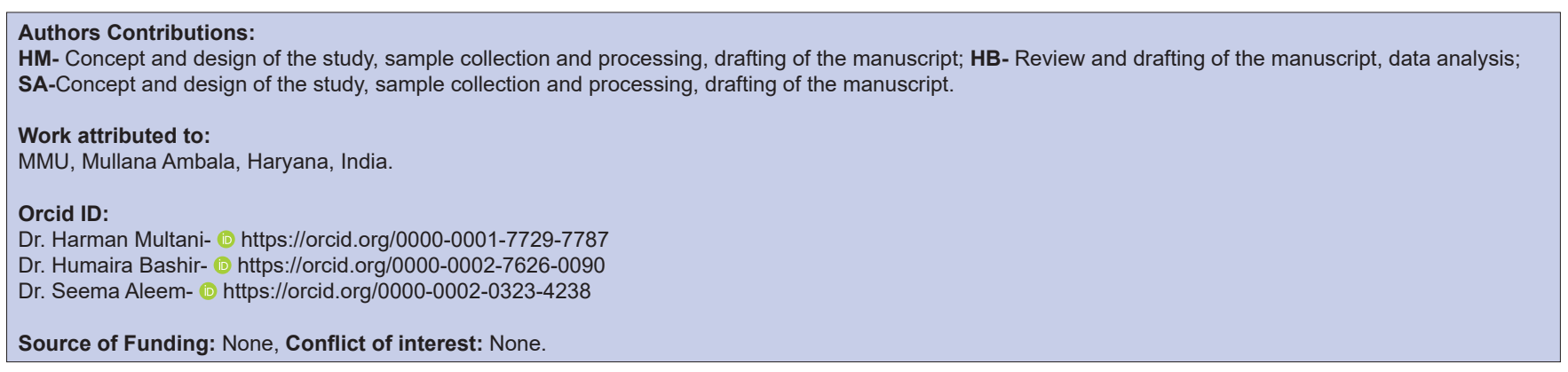

